\title{
Inkjet Printing of Silver Nanowire Networks
}

David J. Finn, Mustafa Lotya and Jonathan N. Coleman*

School of Physics, CRANN and AMBER, Trinity College Dublin, Dublin 2, Ireland

*colemaj@tcd.ie

ABSTRACT: The development of printed electronics will require the ability to deposit a wide range of nano-materials using printing techniques. Here we demonstrate the controlled deposition of networks of silver nanowires in well-defined patterns by inkjet printing from an optimized isopropanol-diethylene glycol dispersion. We find that great care must be taken while producing the ink and during solvent evaporation. The resultant networks have good electrical properties, displaying sheet resistances as low as $8 \Omega / \square$ and conductivities as high as $10^{5} \mathrm{~S} / \mathrm{m}$. Such optimised performances was achieved for line widths of 1-10 mm, and network thicknesses of 0.5-2 $\mu \mathrm{m}$ deposited from $~ 10-20$ passes while using processing temperatures of no more than $110^{\circ} \mathrm{C}$. Thin networks are semi-transparent with DC to optical conductivities of $\sim 40$.

Keywords: Printed electronics, solution processing, nano-materials, network, percolation, silver nanowires, silver nanoink, inkjet fabrication of Ag nanowires. 


\subsection{INTRODUCTION}

The production of low-cost electronic devices will be revolutionized by the ability to print functional materials. Printed electronics involves the additive fabrication of devices on substrates and over large areas at relatively low temperatures. One of the simplest methods is inkjet printing, a non-contact, additive process which can deposit droplets of ink on a substrate in pre-defined patterns. ${ }^{1}$ Devices can be inkjet printed from any material that can be dispersed, suspended or dissolved in a liquid. Examples of advanced materials which have been printed in this way ${ }^{2}$ are polymers/nanocomposites ${ }^{3-6}$ nanoparticles, ${ }^{7-8}$ carbon nanotubes, ${ }^{9-15}$ graphene $^{16-}$ ${ }^{27}$ and most recently $\mathrm{MoS}_{2}$ nanosheets. ${ }^{26,28-30}$ These materials have been used to fabricate a range of devices including photodetectors, ${ }^{26}$ transistors, ${ }^{30-32}$ sensors, ${ }^{5,} 33-34$ light emitting diodes, ${ }^{35}$ supercapacitors, ${ }^{36-37}$ and solar cells. ${ }^{4,38}$

One important application of inkjet printing is the fabrication of conductive elements in printed circuitry. While PEDOT:PSS, ${ }^{39}$ carbon nanotubes ${ }^{13,40}$ and graphene nanosheets ${ }^{16-26}$ have been proposed for use in this space, probably the most successful conductive inks have been based on metallic nanoparticles ${ }^{10,41-45}$ (see SI for literature review table). Inkjet printing of metallic nanoparticles can result in conductive traces with conductivity approaching that of bulk metal. ${ }^{14}$ However, achieving such high conductivities generally requires annealing which can be problematic when working with polymeric substrates. In addition, the annealed structures tend to be dense and so highly optically absorbing. This makes metallic nanoparticles unsuited for printed applications where transparency is required. ${ }^{46}$

Conversely, metallic nanowires, most commonly found as silver nanowires (AgNWs), have recently demonstrated huge potential in transparent electrode applications. ${ }^{46-47}$ Highly conductive silver nanowire electrodes can be fabricated at low temperature, over large areas and are extremely flexible. ${ }^{48-50}$ They have demonstrated sheet resistances as low as $5 \Omega / \square$ for 
transparencies of $90 \% .{ }^{50}$ However, while many methods for the deposition of metallic nanowires have been investigated, ${ }^{48-49,51-55}$ direct writing of patterned, high quality silver nanowire networks has proven extremely difficult. To the best of the author's knowledge very few papers have described direct writing of metallic nanowire traces, in this case using inkjet printing. ${ }^{56-58}$ Of the papers that do exist, only one describes the printing of AgNWs in any detail. $^{57}$ This paper described the printing of AgNWs as a minority component in an AgNW/silver nitrate mixture. The printed structures were converted to silver lines by annealing at $200 \mathrm{C}$. While this work is of great interest, we believe it is important to demonstrate and describe the inkjet printing of structures consisting solely of silver nanowires, preferably at temperatures which are low enough to be compatible with plastic substrates. This is extremely challenging as the high aspect ratio of silver nanowires makes it extremely difficult to print without blocking of the nozzle. In addition, the high density of silver means the given suspended nanowire mass fraction is equivalent to a very low volume fraction. This results in a relatively small number of wires deposited per volume of liquid printed leading to difficulties with solvent management.

We believe the ability to print patterned networks of silver nanowires is important as it would allow the fabrication of patterned, flexible structures which are extremely conductive and highly transparent. Critically, this should be achievable at low temperatures, making this method suitable for use with plastic substrates. In this work, we demonstrate inkjet printing of silver nanowire networks on flexible substrates. We outline both the problems and the solutions which must be found to achieve successful printing. We characterise the resulting networks electrically and show them to be promising for use in printed conducting applications.

\subsection{RESULTS AND DISCUSSION}

\subsection{Printing silver nanowires}


Before printing conductive patterns of AgNWs on flexible substrates such as PET, a number of significant problems must be resolved. For example, the as-delivered nanowires must be shortened and the concentration optimised to avoid nozzle congestion; the correct solvent mixture with appropriate rheological properties must be identified to ensure good jetting performance. The combination of drying procedure and required number of print passes must be carefully managed while a post printing anneal, using temperature compatible with the polymeric substrate, is generally employed to obtain conducting networks. We note that detailed information outlining the effect of varying these parameters is given in the supporting information.

The silver nanowires used in this work were purchased from Seashell Technology as a suspension in isopropanol $(5 \mathrm{mg} / \mathrm{ml})$. These wires are coated with a proprietary organic stabilising agent which prevents aggregation of the wires in isopropanol. As a result, all experiments used isopropanol as the main solvent. Scanning electron microscopy showed the suspended nanowires to have an average diameter of $55 \mathrm{~nm}$ and an average length of $8.1 \mu \mathrm{m}$. These wires are relatively rigid and show no entanglement and very little bending. In this work, a Dimatix Materials Printer (nozzle diameter, $\mathrm{a}=21.5 \mu \mathrm{m}$ ) was used. It is well known that printing of nano-materials generally works best when the nano-objects long dimension is less than a/50. However, we reasoned that this criterion might not be as stringent for nanowires due to the possibility of flow induced alignment as the wires passed through the nozzle. We reduced the nanowire length to $2.2 \mu \mathrm{m}$ by sonication-induced $\operatorname{scission}^{59}$ in a low-power ultrasonic bath for $3 \mathrm{hr}$. Trials showed that, even though the length of these wires was larger than $\mathrm{a} / 50$, they could indeed be successfully inkjetted (see supporting information for more details).

However nanowires dispersed in isopropanol alone do not inkjet well mainly because the solution viscosity is too low. This manifests itself in the formation of satellite droplets and 
generally poor printing. We found that dilution of the isopropanol suspension and the addition of diethylene glycol, such that the total nanowire concentration was $0.85 \mathrm{mg} / \mathrm{ml}$ and the IPA:DEG volumetric ratio was $0.85: 0.15$, gave the best printing performance without the production of any satellite droplets. We note that compositions of 0.90:0.10 and 0.80:0.20 did not print well (see supporting information for more details). We measured the room temperature viscosity and surface tension of this optimised suspension to be $\eta=4.6 \pm 0.3 \mathrm{mPas}$ and $\gamma=28 \mathrm{~mJ} / \mathrm{m}^{2}$ using an Anton-Paar MCR 301 Rheometer (Parallel Plate PP50 geometry) and a TBS Torsion Balance Model OS, respectively. This results in an inverse Ohnesorge number, ${ }^{60}$ $Z=\sqrt{\gamma \rho a} / \eta \approx 4.7\left(\rho \approx 800 \mathrm{~kg} / \mathrm{m}^{3}\right.$ is the liquid density), well within the accepted range for inkjet printing: $1<\mathrm{Z}<14 .{ }^{25}$ The non-optimised compositions $(0.90: 0.10$ and 0.80:0.20) had $\mathrm{Z}$ values approximately $25 \%$ above and below the optimised composition respectively. We note that the nanowire suspension was heated to $40^{\circ} \mathrm{C}$ within the actuation chamber to facilitate subsequent drying. However, the resultant reduction in $\eta$ is certainly not enough to shift $\mathrm{Z}$ out of the required range. Interestingly, we found that if the concentration was increased above $1 \mathrm{mg} / \mathrm{ml}$, printing was impossible due to rapid clogging of the nozzles (see supporting information for more details).

A significant problem here is controlling solvent evaporation during and after deposition. Because of the high density of silver, the solids volume fraction at the optimised concentration $(0.85 \mathrm{mg} / \mathrm{ml})$ is considerably lower than would be the case for a low density material such as graphene. As such, for a given volume of nanowires deposited on a surface the solvent volume which must be removed is proportionally larger. To evaporate the solvent, we initially tried heating the substrate on a vacuum platen at $60{ }^{\circ} \mathrm{C}$ during and after printing. However, we found that this resulted in insulating networks, perhaps due to removal of the organic coating, leading to nanowire oxidation in the heated liquid. To address this we reduced 
the platen temperature to $40{ }^{\circ} \mathrm{C}$, achieving reasonable solvent evaporation (see supporting information for more details). However, to increase the network thickness, it is necessary to increase the number of print passes, N. We found that for more than 10 print passes, solvent tended to bleed out from the printed lines, flooding the substrate. This resulted in very poor quality, non-uniform printed lines.

We found we could address this problem by introducing an intermediate drying step during a print session (see supporting information for more details). After 10 print-passes, the printed PET substrate was placed into a vacuum oven at a temperature of $110{ }^{\circ} \mathrm{C}$ and pumped down to a pressure of $0.1 \mathrm{mPa}$ for 30 minutes to slowly evaporate the solvent. In a next step, the substrate was taken out of the oven and realigned on the printer platen. The printing session was resumed with subsequent printing on top of the oven dried traces. This process was repeated every 10 passes and largely eliminated the bleeding of the solvent vehicle for print runs with large number of passes. After completing the printing session, the inkjet-printed substrate was placed again in the oven under vacuum and annealed at $110{ }^{\circ} \mathrm{C}$ for a period not exceeding two hours. We note that this final drying step also acted as an annealing step to decrease the inter-junction resistivity of the overlapping nanowires and increase the DC conductivity of the network. ${ }^{52}$

In this way it is possible to print good quality lines features and patterns from silver nanowires as shown in figure $1 \mathrm{~A}$. It is very easy to control the lateral dimensions of the features, for example the length and width of printed lines (figure 1B). As indicated above, the thickness of the printed features can be controlled via the number of print passes, $\mathrm{N}$. We investigate the uniformity of such printed features using scanning electron microscopy. Shown in figure $1 \mathrm{C}$ is an SEM image of a relatively thin silver nanowire network $(\mathrm{N}=12)$, which demonstrates the uniformity of these printed features. Figure 1D shows in image of a thicker network $(\mathrm{N}=80)$ 
focusing on the edge region. This image highlights that these networks consist of largely random arrays of rigid AgNWs. By examining freeze-fractured nanowire networks edge-on (figure 1E), is possible to estimate the network thickness. We performed this analysis for a number of networks prepared with different numbers of print passes. As shown in figure $1 \mathrm{~F}$ the measured thickness clearly scales with N. However, unlike the linear behaviour previously observed for inkjet printed graphene networks, ${ }^{22,26}$ here we observe the network thickness to scale very clearly with the square of the number of passes. Fitting this data gives the relationship:

$$
t(\mathrm{~nm})=3.16 N^{1.95 \pm 0.16}
$$

which allows us to estimate the thickness for a given number of passes assuming the suspension concentration and printing conditions remain constant. It is not clear why this relationship should be superlinear, however we suggest it may be related to the issues associated with solving drying described above.

Once this process optimisation has been achieved, it is possible to directly deposit patterned networks. To demonstrate this we printed out the Trinity College Dublin crest as shown in figure $1 \mathrm{G}$. It is clear from this image that the accuracy and resolution achievable using this process is reasonably good, at least at length scales above $\sim 100 \mu \mathrm{m}$ (see below).

\subsection{Network uniformity}

In order to assess the thickness uniformity of the printed AgNW networks, we performed transmission scanning ${ }^{48}$ on a network produced with $\mathrm{N}=20$ and $\mathrm{w}=2 \mathrm{~mm}$. In this method, the local transmittance of the sample is measured with a pixel size of $\sim 6 \mu \mathrm{m}$. Scanning was performed on network deposited on a PET substrate. The resultant transmittance measurements can then be plotted as a map to form an image such as the one shown in figure 
2A. In this image, the printed line is clearly apparent. It can be seen that the edge of the line is somewhat rough, with a line-edge roughness of 100-200 $\mu \mathrm{m}$. In addition, horizontal striations can be seen in the print pattern which are aligned in the raster directions (x-axis). These are known as swathe edges in the printing industry and occur along the direction parallel to the ink head raster direction. ${ }^{61-62}$ These features have also been seen in inkjet printed graphene networks ${ }^{26}$ and were present for all $\mathrm{AgNW}$ networks produced in this work.

The measured transmittance can be transformed to absorbance, A, using $T=10^{-A}$. The absorbance is of interest as we expect it to be proportional to the local thickness ${ }^{26}$ and so a metric for network uniformity. Shown in figure $2 \mathrm{~B}$ is a plot of local absorbance as a function of horizontal position (X) along the red dashed line in A. This profile is clearly box-like with a baseline of $\sim 0.1$, corresponding to the absorbance of the PET substrate. The printed line rises from edge to maximum thickness over $300-400 \mu \mathrm{m}$ and is relatively uniform over the central $1.8 \mathrm{~mm}$. By subtracting off the PET absorbance, it is possible to transform the data in figure 2B to represent the $\mathrm{AgNW}$ network transmittance as shown in figure $2 \mathrm{C}$. This plot shows the mean visible transmittance of the $\mathrm{N}=20$ line to be $\sim 50 \%$. Shown in figure $2 \mathrm{D}-\mathrm{E}$ are crosssection data for measured absorbance and AgNW network transmittance along the vertical blue dashed line in figure 2A. These data are similar to the central portion of the lines shown in figure 2B-C, although the data does appear noisier. However, closer examination (insets) shows the excess noise to be largely due to the striations shown in figure $2 \mathrm{~A}$. The inset data shows the thickness variation due to the striations to be at most $25 \%$ of the mean thickness.

We can examine the uniformity of the networks directly by extracting data for the network absorbance (i.e. the measured absorbance minus the PET absorbance) for a large number of pixels in the regions marked by the red and blue boxes in figure A. The blue box represents a region $30 \mu \mathrm{m}$ wide by $12 \mathrm{~mm}$ (9990 pixels) deep while the red box represents a 
region $1.5 \mathrm{~mm}$ wide by $30 \mu \mathrm{m}$ deep (1255 pixels). This data has been plotted as histograms in figure $2 \mathrm{~F}$ and $\mathrm{G}$ for the red and blue boxes respectively. These histograms represent the distributions in local absorbance, and so network thicknesses, in the horizontal and vertical directions respectively. In both cases they are relatively narrow indicating the lines to be reasonably uniform. However, the distribution in figure $2 \mathrm{G}$ is clearly slightly broader due to the presence of the horizontal striations shown in figure 2A. Statistical analysis shows the ratio of distribution standard deviation to mean to be $<10 \%$ for both distributions.

\subsection{Electrical Properties of Silver Nanowire Networks}

It is very important that these networks show reasonable electrical properties. To test this, we sprayed three sets of silver nanowire traces, independently varying the length, $\mathrm{L}$, the width, w, and the number of passes, N. Shown in figure $3 \mathrm{~A}$ is data for the resistance as a function of trace length $(\mathrm{N}=20, \mathrm{w}=2 \mathrm{~mm})$ for two independent sets of lines. The resistance scales linearly with length up to $\mathrm{L}=40 \mathrm{~mm}$ in a manner consistent with a sheet resistance of $R_{S}=19 \Omega / \square$ (dashed line). For longer traces the resistance is higher than one would expect. We associate this with the flooding described above which was observed to occur for long traces. Such flooding causes nanowires to bleed out of the traces leading to significant non-uniformity and reduced electrical performance. We also measured the resistance as a function of trace width, w, as shown in figure 3B for two independent sets of lines. From Ohms law, one would expect the resistance to scale inversely with width. However, this data deviates considerably from the expected behaviour, showing linear behaviour only in a narrow range between 1 and $10 \mathrm{~mm}$. For widths above $10 \mathrm{~mm}$, the resistance is higher than expected. This can be explained by solvent flooding and the resultant non-uniformities. The resistances at widths below $1 \mathrm{~mm}$ are also higher than expected for an Ohmic material. We associate this phenomenon with line- 
edge-roughness. We assume the traces have a minimum width $w_{0}$ and roughness on either side of the trace with amplitude $\Delta \mathrm{w}$. Then, the overall average width is $w=w_{0}+\Delta w$, which is equal to the perceived width. However, the width which limits the electrical conductivity is wo. This means that the most appropriate expression for the line resistance is

$$
R=\frac{R_{s} L}{w_{0}}=\frac{R_{s} L}{w-\Delta w}
$$

We have fit this expression to the data for $\mathrm{w}<10 \mathrm{~mm}$, finding very good agreement for $\mathrm{R}_{\mathrm{s}}=8$ $\Omega / \square$ and $\Delta \mathrm{w}=180 \mu \mathrm{m}$. Optical microscopy (not shown) confirms the roughness of the line edges to be of order of hundred microns, consistent with the data in figure $2 \mathrm{~A}$.

As described above, the thickness of the network is controlled by the number of print passes, N. Shown in figure $3 \mathrm{C}$ is a graph of the measured resistance as a function of $\mathrm{N}$ for two independent sets of lines $(\mathrm{L}=20 \mathrm{~mm}, \mathrm{w}=2 \mathrm{~mm})$. This data shows a very high resistance for thin lines, corresponding to a low number of passes. The resistance appears to saturate somewhat for $\mathrm{N}>20$. To understand this data, it is necessary to convert $\mathrm{N}$ into thickness, $\mathrm{t}$, using equation 1 and then resistance into conductivity using

$$
\sigma=\frac{L}{R w t}
$$

The resulting conductivity data is plotted versus network thickness in figure 3D. This data clearly shows two regimes, one for $\mathrm{t}>500 \mathrm{~nm}$ where conductivity is invariant with thickness and for $\mathrm{t}<500 \mathrm{~nm}$ whether conductivity falls sharply with decreasing thickness. This data is consistent with a number of previous observations relating to the electrical conductivity of nanostructures networks. It is well known that, for networks of nano-conductors such as silver nanowires, no conductivity is observed below a critical network thickness which is known as 
the percolation threshold. ${ }^{63}$ This threshold corresponds to the formation of the first conductive path. Above the percolation threshold, $t_{c}$, the electrical conductivity scales with the thickness as $^{63}$

$$
\sigma \propto\left(t-t_{c}\right)^{n}
$$

where $\mathrm{n}$ is the percolation exponent. We fit this equation to the data in the thickness range between 250 and $500 \mathrm{~nm}$, finding good agreement using the fit constants $t_{c}=255 \mathrm{~nm}$ and $\mathrm{n}=1.1$. While this value of $\mathrm{n}$ is close to that expected for two-dimensional networks (i.e. $\mathrm{n}=1.3$ ),${ }^{63}$ this value of $t_{c}$ is unexpectedly high as we shall discuss below. It is well known that, above a certain thickness, the electrical properties of nano structured networks become thickness independent, displaying a bulk-like conductivity. ${ }^{64-65}$ This behaviour can be seen in figure $3 \mathrm{D}$ for thicknesses greater than $500 \mathrm{~mm}$. In this case the data is consistent with the bulk-like conductivity of $3 \times 10^{4}$ $\mathrm{S} / \mathrm{m}$. In addition, there is some evidence for reduction in conductivity at very high thicknesses due to flooding effects. However, we note that there may be some problems with this data and its interpretation. The percolation threshold is much higher than expected. Previous papers have shown the percolation thresholds for silver nanowire networks to be below 100 nanometres. ${ }^{48}$ In addition, the transformation from percolative to bulk-like behaviour occurs here at $500 \mathrm{~nm}$. In previous work this transformation has been observed to occur at thicknesses of roughly 3 times the nanowire diameter. ${ }^{64-65}$ Thus, we would expect this transformation to occur for network thicknesses of approximately $150 \mathrm{~nm}$. Taken together, this suggested either our thicknesses are overestimated or inkjet printed nano networks are morphologically different from those previously studied. If the first suggestion is true then this means that the conductivities quoted here are under-estimates. Further work is required to clarify this point.

We can test the consistency and reproducibility of the electrical properties of these networks by plotting R versus $\mathrm{L} / \mathrm{wt}$ (i.e. as per equation 3) for all data on a single plot as shown 
in figure 3E. We find the data to be roughly consistent and follow the same trend. For an Ohmic material, such a graph should be purely linear. However, here we only observe linearity over a limited range. This behaviour is indicated by the dashed lines which show the network conductivities to vary between $\sigma=2 \times 10^{4} \mathrm{~S} / \mathrm{m}$ and $\sigma=10^{5} \mathrm{~S} / \mathrm{m}$ in the Ohmic (bulk-like) regime. These conductivities are relatively low for silver nanowire networks. For example, in our lab we have routinely prepared nanowire networks by both spraying and vacuum filtration with conductivities as high as $5 \times 10^{6} \mathrm{~S} / \mathrm{m} .{ }^{48-49}$ Other authors have produced even more conductive networks. ${ }^{50,66}$ Part of the reason for the low observed conductivity is associated with the reduced length of the wires. The conductivity of nanowire networks is known to scale at least linearly ${ }^{67}$ with nanowire length with some reports suggesting super linear behaviour. ${ }^{68}$ In most cases in the literature the mean wire length exceeds $5 \mu \mathrm{m}$ and may be as high as many tens of microns ${ }^{69}$ far greater than the mean length of $2.2 \mu \mathrm{m}$ used here. In addition, as described above, the conductivity of our networks may be somewhat underestimated due to uncertainties in the network thickness. However, it may be that the conductivity of inkjet printed nanowire networks is lower than that of networks produced by other means due to morphological differences. For example, work in our lab has shown that inkjet printed networks of graphene nanosheets display conductivity about 10 times lower than networks produced by vacuum filtration. ${ }^{26}$ Further work is required to identify if the inkjet printed network morphology is resulting in reduced conductivities, perhaps due to disruption of junction annealing. Finally, it is worth noting that the deviations from linearity for both low and high values of L/wt are due to flooding/bleeding and the effect of percolation/line edge roughness, respectively.

It is important to put these results in context. For printed electronics applications it would be critically important to print features with high conductivity while minimising processing temperature. Shown in figure $3 \mathrm{~F}$ is a reasonable, but not exhaustive, attempt to summarise data from the literature by plotting the maximum conductivity achieved versus the 
maximum annealing temperature used. We have limited data to inkjet-printed structures and categorised in terms of the materials used: Graphene, ${ }^{16-26}$ nanotubes ${ }^{13,40}$ and nanoparticles of silver ${ }^{10,41-44}$ and gold. ${ }^{45}$ We find the majority of inks to demonstrate lower conductivity and/or higher processing temperatures than those reported here. From this cohort, only two inks, fabricated from nanotubes ${ }^{40}$ and silver nanoparticles ${ }^{43}$ show both higher conductivity coupled with lower processing temperatures. However, we are certain the conductivity of the printed nanowire networks reported here can be improved significantly by optimising the thermal annealing process or, for example, by optical welding of junctions. ${ }^{70}$

Finally, we note that for transparent conductor applications, what is important is not the electrical conductivity but the ratio of electrical to optical conductivity, ${ }^{64} \sigma_{D C} / \sigma_{O p}$ (the optical conductivity is a measure of how strongly a material absorbs light and is proportional to the absorption coefficient). This parameter controls the relationship between transmittance and sheet resistance via:

$T=\left(1+\frac{Z_{0}}{2 R_{s}} \frac{\sigma_{O p}}{\sigma_{D C}}\right)^{-2}$

where $Z_{0}=377 \Omega$ is the impedance of free space. ${ }^{64}$ According to figures 2 and 3 , for the $N=20$ networks, the mean sheet resistance is $\sim 10 \Omega / \square$ while the transmittance estimated from the scanner is $\sim 50 \%$. Using equation 5 , this means the networks produced here are described by $\sigma_{D C} / \sigma_{O p} \sim 40$. Again using equation 5, we can predict that such networks would display $\mathrm{R}_{\mathbb{S}} \sim 90$ $\Omega / \square$ at a thickness suitable to give $\mathrm{T}=90 \%$. This sort of performance is reasonably good for nanostructured transparent conductors in general ${ }^{64}$ but relatively poor for transparent nanowire networks which can display $\sigma_{D C} / \sigma_{O p}>400\left(\operatorname{ref}^{64}\right)$ and $\mathrm{R}_{\mathrm{s}}(\mathrm{T}=90 \%)<10 \Omega / \square .{ }^{50}$ The $\mathrm{DC}$ to optical conductivity ratio is low primarily because of the low DC conductivity of these 
networks $\left(2-10 \times 10^{4} \mathrm{~S} / \mathrm{m}\right)$. To test this, we estimate the optical conductivity (using $\left.\sigma_{D C} / \sigma_{O p}\right)$ to be $\sigma_{\mathrm{Op}}=500-2500 \mathrm{~S} / \mathrm{m}$ which is relatively close but somewhat below to the range previously reported 3800-6500 S/cm. ${ }^{48-49}$ Because $\sigma$ Op is lower than expected, the low value of $\sigma_{D C} / \sigma_{O P}$ must be due to the to the relatively low value of $\sigma_{\mathrm{DC}}$. The reasons for this have been described above.

\subsection{CONCLUSION}

In summary, we have demonstrated inkjet printing of networks of silver nanowires, from optimized ispropanol-diethylene glycol dispersions. We have identified the main challenges to achieving this, resolving them one by one. The resultant networks are reasonably uniform and have good electrical properties. The highest sheet resistance observed was $8 \Omega / \square$ while the highest conductivity was $10^{5} \mathrm{~S} / \mathrm{m}$. Such optimised performances was achieved for line widths of 1-10 mm, and network thicknesses of 0.5-2 $\mu \mathrm{m}$ deposited from 10-20 passes while using processing temperatures of no more than $110{ }^{\circ} \mathrm{C}$. While further improvements are necessary, this data is already competitive with most of the published data on printed nanomaterials.

We believe this work is important as one of the first steps toward the ability to print highly conductive, transparent, patterned networks of metallic nanowires. Future work will see the improvement of the network conductivity, perhaps by the introduction of low-temperature sintering processes to weld nanowire junctions together. This will lead to inkjet printed nanowires becoming an important addition to the palette of materials already available to the printed electronics community. 


\subsection{METHODS}

Silver nanowires were purchased from Seashell Technology as a suspension in isopropanol at the concentration of $5 \mathrm{mg} / \mathrm{ml}$. Ultrasonic induced scission of the silver nanowires to reduce their length was carried out in a low power sonic bath (Branson 1510E-MT) operating at a frequency of $42 \mathrm{kHz} \pm 5 \%$ and a rated power of 70 watts.

In this work, a Dimatix Materials Printer 2800 was used. This laboratory inkjet printer uses a single print head having 16 ejection nozzles (nozzle diameter $=21.5 \mu \mathrm{m})$ spaced $254 \mu \mathrm{m}$ apart, driven by piezoelectric elements jetting 10 picoliter droplets, and fed with a solvent-based ink from a fluid bag encased in a plastic housing attached to the print head. The print head angle was $4.5^{\circ}$ while a typical drop spacing was $20 \mu \mathrm{m}$ with a $50 \%$ degree of overlap. The drop frequency was $5 \mathrm{kHz}$ while the jetting waveform parameters are shown in the SI. We note that clogging of the printhead did not occur if the jetting parameters described above were applied. However, the appropriate cleaning cycles were important to avoiding nozzle clogging. It was observed that purging of the print head and ink tank following use greatly extended the useable life of the printhead. For example, preventing drying of the nanowires on the printhead was particularly important. It was found that with regular purging during printing and careful cleaning following printing, the printhead could be used for several days, and up to 10 days in some cases, without any degradation in print performance. The substrate used was a coated PET designed for printing of silver inks (Mitsubishi NB-TP-3GU100). Solvent evaporation was managed as described in the text. The inkjet printer used in this study defines patterns based on an XY grid coordinate system. Patterns are programmed as bitmap images. For this work the "length" and "width" were varied independently by changing the pattern shape, drawing a series of adjacent lines with the following variables: constant length (in Y direction) 
with varied width (in $\mathrm{X}$ direction), constant width in (X direction) with varied length in (Y direction). The drop volume, frequency and spacing were held constant throughout.

Profile uniformity analysis of a silver nanowire network after 20 print passes was made using an Epson Perfection V700 photo flatbed transmission scanner with a bit depth of 48-bits per pixel and a spatial resolution of 4800 dpi (see SI for more information). Scanning electron microscopy was performed using a Carl Zeiss Ultra Plus scanning electron microscope. The electrical characterization was performed using a Keithley 2400 source meter using the four probe technique.

We acknowledge support from the SFI-funded AMBER research centre (SFI/12/RC/2278). 

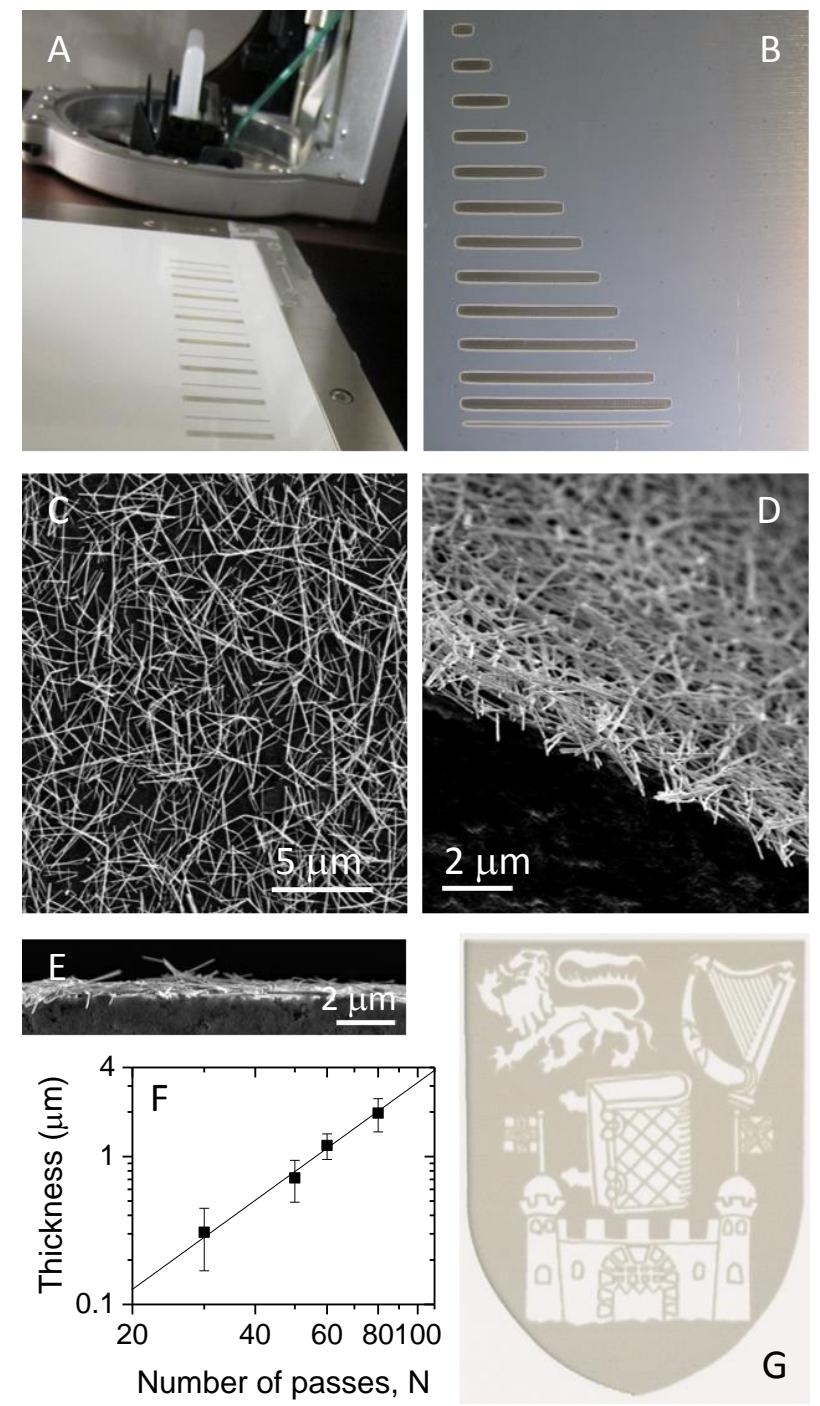

Figure 1: Inkjet printed silver nanowire networks. A) Inkjet printing silver nanowire lines on a coated PET substrate which has been aligned with index pins on the printer's heated platen. B) Printed lines of different lengths $(\mathrm{w}=2 \mathrm{~mm}, \mathrm{~N}=20)$. C) An SEM image of a relatively thin silver nanowire network ( $\mathrm{N}=12$ passes). D-E) SEM images of the edges of thick nanowire networks (D: $\mathrm{N}=80$ passes and $\mathrm{E}: \mathrm{N}=50$ passes). F) Network thickness, as measured by SEM, as a function of number of passes. G) Inkjet printed nanowire network in the shape of the Trinity College Dublin crest. The width of this crest is $\sim 4 \mathrm{~cm}$. 

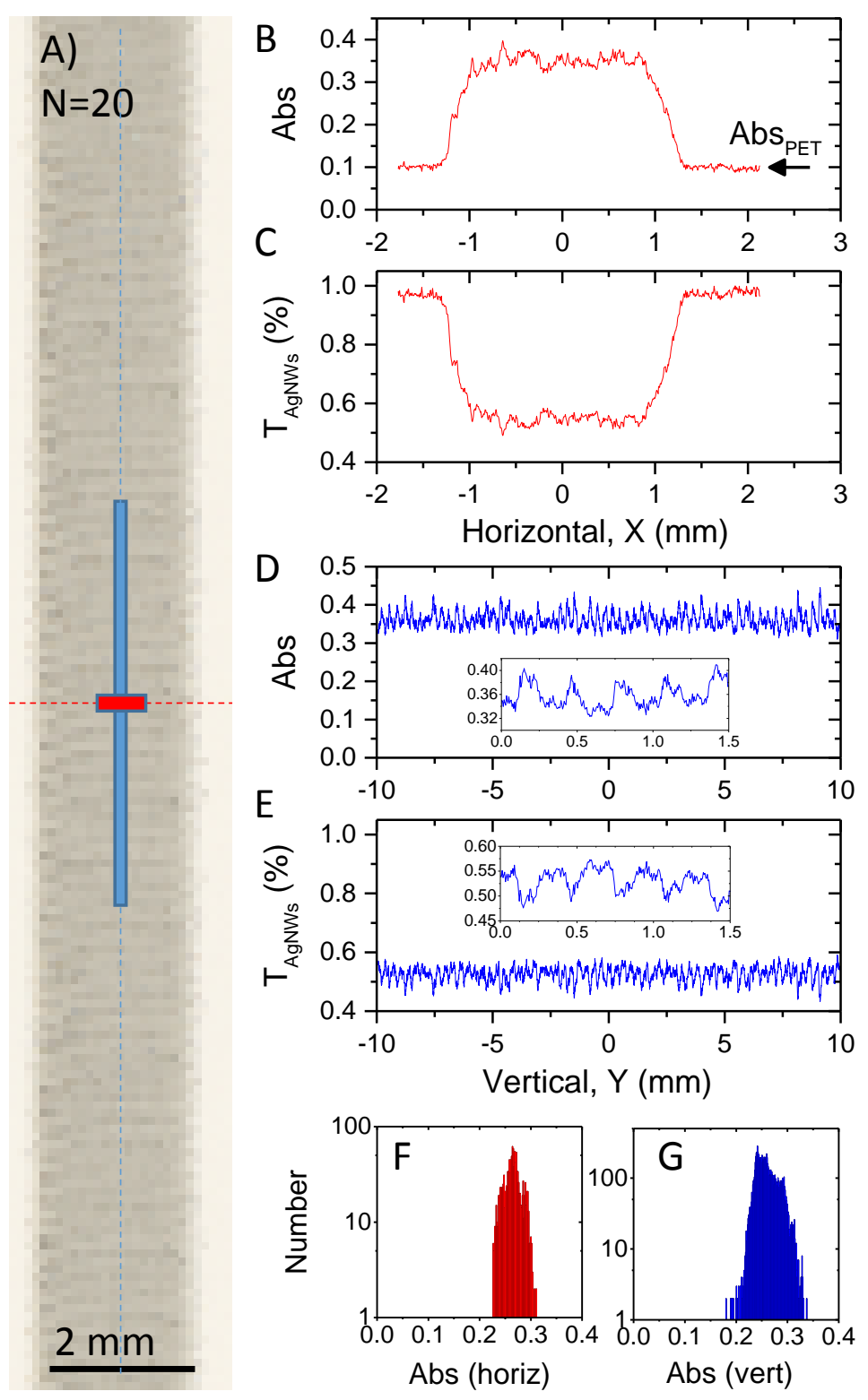

Figure 2: A) A transmission scanned image of an inkjet printed $(\mathrm{N}=20, \mathrm{w}=2 \mathrm{~mm}) \mathrm{AgNW}$ line on PET. The print raster direction was horizontal. Note the striations parallel to the raster direction. B) Measured absorbance profile (extracted from the transmission scan) along a horizontal cross-section (red dashed line in A). Note that the absorbance does not fall to zero beyond the edge of the line as the PET substrate was not fully transparent but displayed APET 0.1 (TPET 80\%). C) The transmittance of the AgNW network (extracted from B by subtracting the PET absorbance) along a horizontal cross section. D-E) Measured absorbance (D) along a vertical cross-section (blue dashed line in A) along with the derived AgNW 
network transmittance (E). The insets in D and E show variations in both absorbance and transmittance along the vertical cross-section associated with the striations seen in A). These striations appear with a frequency of 3.1/mm. F-G) Histograms of AgNW network absorbances (i.e. measured abs minus PET abs) extracted from regions of the image in A schematically shown by the red $(\mathrm{F})$ and blue $(\mathrm{G})$ rectangles.
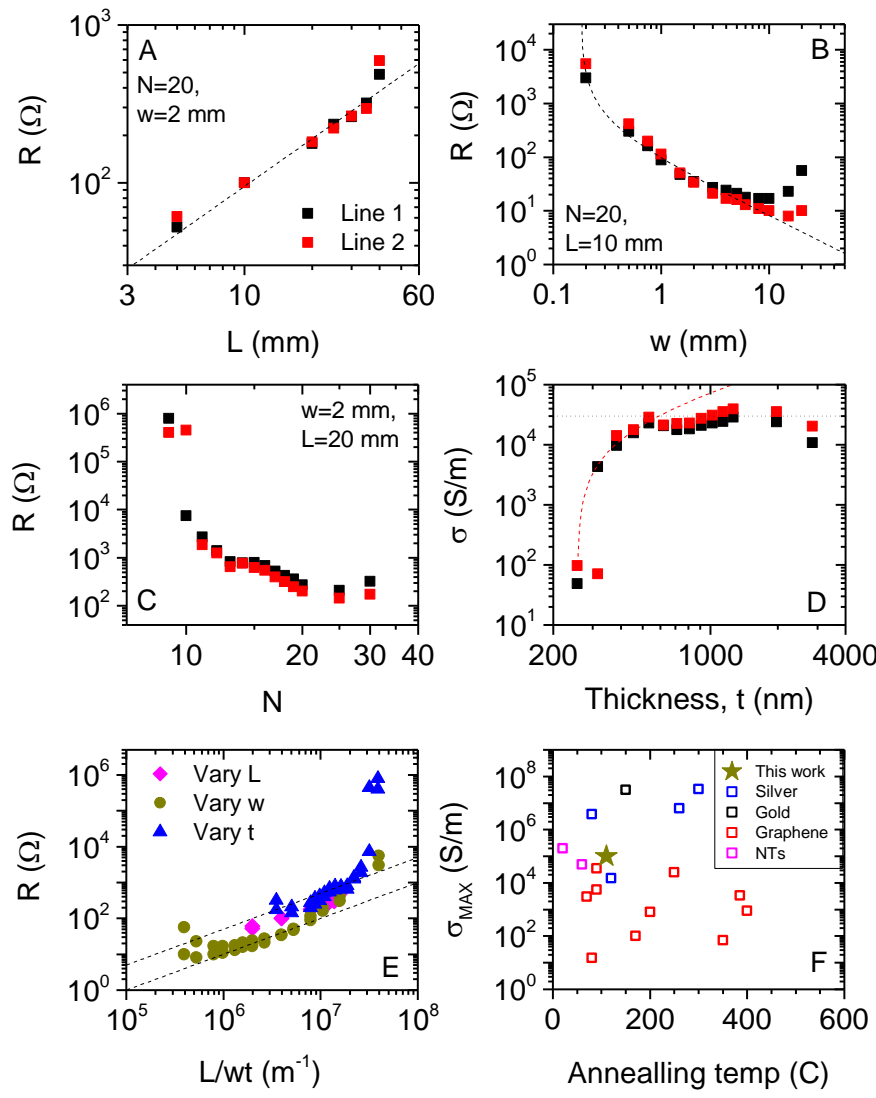

Figure 3: Electrical properties of inkjet printed silver nanowire networks. A-C) Electrical resistance of lines of inkjet printed nanowires plotted as a function of A) line length (inter- 
electrode distance), B) line width and C) number of print passes. In A) the dashed line represents linearity and is consistent with $R_{s}=19 \Omega / \square$ while in B) the dashed line represents a fit to equation 2 with $\mathrm{R}_{\mathrm{s}}=8 \Omega / \square$ and $\Delta \mathrm{w}=180 \mu \mathrm{m}$. D) Electrical conductivity plotted as a function of line thickness. The dashed line illustrates percolative behaviour while the dotted line illustrates bulk-like behaviour. E) Electrical resistance plotted versus L/wt, were $t$ is calculated from $\mathrm{N}$ using equation 1 . In this plot a bulk-like material obeying Ohms law will display linear behaviour as indicated by the dashed lines (consistent with $\sigma=2 \times 10^{4} \mathrm{~S} / \mathrm{m}$ and $\sigma=10^{5} \mathrm{~S} / \mathrm{m}$ for the upper and lower curves respectively). In A-E data is given for two independent sets of lines. F) Comparison with literature data. Conductivity plotted versus annealing temperature for lines printed from graphene, carbon nanotube, silver and gold nanoparticle based inks.

Supporting Information Available: This includes a detailed description of the printing optimisation. This information is available free of charge via the Internet at http://pubs.acs.org/.

\section{References}

1. Hutchings, I. M.; Martin, G. D., Inkjet Technology for Digital Fabrication. John Wiley \& Sons: Chichester, West Sussex, 2013.

2. Kamyshny, A.; Magdassi, S., Conductive Nanomaterials for Printed Electronics. Small 2014, $103515-3535$.

3. Yan, H.; Chen, Z. H.; Zheng, Y.; Newman, C.; Quinn, J. R.; Dotz, F.; Kastler, M.; Facchetti, A., A High-Mobility Electron-Transporting Polymer for Printed Transistors. Nature 2009, 457 679-U1.

4. $\quad$ Del Mauro, A. D.; Diana, R.; Grimaldi, I. A.; Loffredo, F.; Morvillo, P.; Villani, F.; Minarini, C., Polymer Solar Cells with Inkjet-Printed Doped-Pedot: Pss Anode. Polym. Compos. 2013, 34 1493-1499.

5. Crowley, K.; Morrin, A.; Shepherd, R. L.; Panhuis, M. I. H.; Wallace, G. G.; Smyth, M. R.; Killard, A. J., Fabrication of Polyaniline-Based Gas Sensors Using Piezoelectric Inkjet and Screen Printing for the Detection of Hydrogen Sulfide. (IEEE) Sensors J. 2010, 101419 1426. 
6. Mire, C. A.; Agrawal, A.; Wallace, G. G.; Calvert, P.; Panhuis, M. I. H., Inkjet and Extrusion Printing of Conducting Poly(3,4-Ethylenedioxythiophene) Tracks on and Embedded in Biopolymer Materials. J. Mater. Chem. 2011, 21 2671-2678.

7. Wood, V.; Panzer, M. J.; Chen, J. L.; Bradley, M. S.; Halpert, J. E.; Bawendi, M. C.; Bulovic, V., Inkjet-Printed Quantum Dot-Polymer Composites for Full-Color Ac-Driven Displays. Adv. Mater. 2009, 21 2151-2155.

8. Lee, H. H.; Chou, K. S.; Huang, K. C., Inkjet Printing of Nanosized Silver Colloids. Nanotechnology 2005, 16 2436-2441.

9. Takagi, Y.; Nobusa, Y.; Gocho, S.; Kudou, H.; Yanagi, K.; Kataura, H.; Takenobu, T., Inkjet Printing of Aligned Single-Walled Carbon-Nanotube Thin Films. Appl. Phys. Lett. 2013, 102143107.

10. Kim, T.; Song, H.; Ha, J.; Kim, S.; Kim, D.; Chung, S.; Lee, J.; Hong, Y., Inkjet-Printed Stretchable Single-Walled Carbon Nanotube Electrodes with Excellent Mechanical Properties. Appl. Phys. Lett. 2014, 104113103.

11. Sajed, F.; Rutherglen, C., All-Printed and Transparent Single Walled Carbon Nanotube Thin Film Transistor Devices. Appl. Phys. Lett. 2013, 103143303.

12. Beecher, P.; Servati, P.; Rozhin, A.; Colli, A.; Scardaci, V.; Pisana, S.; Hasan, T.; Flewitt, A. J.; Robertson, J.; Hsieh, G. W.; Li, F. M.; Nathan, A.; Ferrari, A. C.; Milne, W. I., Ink-Jet Printing of Carbon Nanotube Thin Film Transistors. J. Appl. Phys. 2007, 102025005. 13. Song, J.-W.; Kim, J.; Yoon, Y.-H.; Choi, B.-S.; Kim, J.-H.; Han, C.-S., Inkjet Printing of Single-Walled Carbon Nanotubes and Electrical Characterization of the Line Pattern. Nanotechnology 2008, 19095702.

14. Shimoni, A.; Azoubel, S.; Magdassi, S., Inkjet Printing of Flexible High-Performance Carbon Nanotube Transparent Conductive Films by "Coffee Ring Effect". Nanoscale 2014, 6 11084-11488.

15. Small, W. R.; Panhuis, M. I. H., Inkjet Printing of Transparent, Electrically Conducting Single-Waited Carbon-Nanotube Composites. Small 2007, 3 1500-1503.

16. Dua, V.; Surwade, S. P.; Ammu, S.; Agnihotra, S. R.; Jain, S.; Roberts, K. E.; Park, S.; Ruoff, R. S.; Manohar, S. K., All-Organic Vapor Sensor Using Inkjet-Printed Reduced Graphene Oxide. Angew. Chem., Int. Ed. 2010, 49 2154-2157.

17. Huang, L.; Huang, Y.; Liang, J. J.; Wan, X. J.; Chen, Y. S., Graphene-Based Conducting Inks for Direct Inkjet Printing of Flexible Conductive Patterns and Their Applications in Electric Circuits and Chemical Sensors. Nano Research 2011, 4 675-684.

18. Kong, D.; Le, L. T.; Li, Y.; Zunino, J. L.; Lee, W., Temperature-Dependent Electrical Properties of Graphene Inkjet-Printed on Flexible Materials. Langmuir 2012, 28 13467-13472. 19. Le, L. T.; Ervin, M. H.; Qiu, H. W.; Fuchs, B. E.; Lee, W. Y., Graphene Supercapacitor Electrodes Fabricated by Inkjet Printing and Thermal Reduction of Graphene Oxide. Electrochem. Commun. 2011, 13 355-358.

20. Li, J.; Ye, F.; Vaziri, S.; Muhammed, M.; Lemme, M. C.; Ostling, M., Efficient Inkjet Printing of Graphene Adv. Mater. 2013, 25 3985-3992.

21. Lim, S.; Kang, B.; Kwak, D.; Lee, W. H.; Lim, J. A.; Cho, K., Inkjet-Printed Reduced Graphene Oxide/Poly(Vinyl Alcohol) Composite Electrodes for Flexible Transparent Organic Field-Effect Transistors. J. Phys. Chem. C 2012, 116 7520-7525.

22. Secor, E. B.; Prabhumirashi, P. L.; Puntambekar, K.; Geier, M. L.; Hersam, M. C., Inkjet Printing of High Conductivity, Flexible Graphene Patterns. J. Phys. Chem. Lett. 2013, 4 1347-1351.

23. Shin, K. Y.; Hong, J. Y.; Jang, J., Micropatterning of Graphene Sheets by Inkjet Printing and Its Wideband Dipole-Antenna Application. Adv. Mater. 2011, 23 2113-2118.

24. Shin, K. Y.; Hong, J. Y.; Jang, J., Flexible and Transparent Graphene Films as Acoustic Actuator Electrodes Using Inkjet Printing. Chem. Commun. 2011, 47 8527-8529. 
25. Torrisi, F.; Hasan, T.; Wu, W. P.; Sun, Z. P.; Lombardo, A.; Kulmala, T. S.; Hsieh, G. W.; Jung, S. J.; Bonaccorso, F.; Paul, P. J.; Chu, D. P.; Ferrari, A. C., Inkjet-Printed Graphene Electronics. ACS Nano 2012, 6 2992-3006.

26. Finn, D. J.; Lotya, M.; Cunningham, G.; Smith, R. J.; McCloskey, D.; Donegan, J. F.; Coleman, J. N., Inkjet Deposition of Liquid-Exfoliated Graphene and Mos2 Nanosheets for Printed Device Applications. J. Mater. Chem. C 2014, 2 925-932.

27. Torrisi, F.; Coleman, J. N., Electrifying Inks with 2d Materials. Nat. Nanotechnol. 2014, 9 738-739.

28. Yao, Y.; Tolentino, L.; Yang, Z.; Song, X.; Zhang, W.; Chen, Y.; Wong, C.-P., HighConcentration Aqueous Dispersions of Mos2. Adv. Funct. Mater. 2013, 23 3577-3583.

29. Zheng, J.; Zhang, H.; Dong, S.; Liu, Y.; Nai, C. T.; Shin, H. S.; Jeong, H. Y.; Liu, B.; Loh, K. P., High Yield Exfoliation of Two-Dimensional Chalcogenides Using Sodium Naphthalenide. Nature Commun. 2014, 52995.

30. Li, J.; Naiini, M. M.; Vaziri, S.; Lemme, M. C.; Östling, M., Inkjet Printing of Mos2. Adv. Funct. Mater. 2014, 24 6524-6531.

31. Sirringhaus, H.; Kawase, T.; Friend, R. H.; Shimoda, T.; Inbasekaran, M.; Wu, W.; Woo, E. P., High-Resolution Inkjet Printing of All-Polymer Transistor Circuits. Science 2000, 290 2123-2126.

32. Zhang, J.; Zhao, Y.; Wei, Z. M.; Sun, Y. M.; He, Y. D.; Di, C. A.; Xu, W.; Hu, W. P.; Liu, Y. Q.; Zhu, D. B., Inkjet-Printed Organic Electrodes for Bottom-Contact Organic FieldEffect Transistors. Adv. Funct. Mater. 2011, 21 786-791.

33. Karuwan, C.; Sriprachuabwong, C.; Wisitsoraat, A.; Phokharatkul, D.; Sritongkham, P.; Tuantranont, A., Inkjet-Printed Graphene-Poly(3,4Ethylenedioxythiophene):Poly(Styrene-Sulfonate) Modified on Screen Printed Carbon Electrode for Electrochemical Sensing of Salbutamol. Sens. Actuators, B 2012, 161 549-555.

34. Monereo, O.; Claramunt, S.; de Marigorta, M. M.; Boix, M.; Leghrib, R.; Prades, J. D.; Cornet, A.; Merino, P.; Merino, C.; Cirera, A., Flexible Sensor Based on Carbon Nanofibers with Multifunctional Sensing Features. Talanta 2013, 107 239-247.

35. Bharathan, J.; Yang, Y., Polymer Electroluminescent Devices Processed by Inkjet Printing: I. Polymer Light-Emitting Logo. Appl. Phys. Lett. 1998, 72 2660-2662.

36. Chen, P. C.; Chen, H. T.; Qiu, J.; Zhou, C. W., Inkjet Printing of Single-Walled Carbon Nanotube/Ruo2 Nanowire Supercapacitors on Cloth Fabrics and Flexible Substrates. Nano Research 2010, 3 594-603.

37. Kossyrev, P., Carbon Black Supercapacitors Employing Thin Electrodes. J. Power Sources 2012, 201 347-352.

38. Hoth, C. N.; Schilinsky, P.; Choulis, S. A.; Brabec, C. J., Printing Highly Efficient Organic Solar Cells. Nano Lett. 2008, 8 2806-2813.

39. Kang, B.; Lee, W. H.; Cho, K., Recent Advances in Organic Transistor Printing Processes. ACS Appl. Mater. Interfaces 2013, 5 2302-2315.

40. Gracia-Espino, E.; Sala, G.; Pino, F.; Halonen, N.; Luomahaara, J.; Mäklin, J.; Tóth, G.; Kordás, K.; Jantunen, H.; Terrones, M.; Helistö, P.; Seppä, H.; Ajayan, P. M.; Vajtai, R., Electrical Transport and Field-Effect Transistors Using Inkjet-Printed Swcnt Films Having Different Functional Side Groups. ACS Nano 2010, 4 3318-3324.

41. Faddoul, R.; Reverdy-Bruas, N.; Blayo, A.; Khelifi, B., Inkjet Printing of Silver NanoSuspensions on Ceramic Substrates - Sintering Temperature Effect on Electrical Properties. Microelectron. Eng. 2013, 105 31-39.

42. Hsien-Hsueh, L.; Kan-Sen, C.; Kuo-Cheng, H., Inkjet Printing of Nanosized Silver Colloids. Nanotechnology 2005, 162436. 
43. Perelaer, J.; de laat, A. W. M.; Hendriks, C. E.; Schubert, U. S., Inkjet-Printed Silver Tracks: Low Temperature Curing and Thermal Stability Investigation. J. Mater. Chem. 2008, 18 3209-3215.

44. Polzinger, B.; Schoen, F.; Matic, V.; Keck, J.; Willeck, H.; Eberhardt, W.; Kueck, H. In Uv-Sintering of Inkjet-Printed Conductive Silver Tracks, Nanotechnology (IEEE-NANO), 2011 11th IEEE Conference on, 15-18 Aug. 2011; 2011; pp 201-204.

45. Huang, D.; Liao, F.; Molesa, S.; Redinger, D.; Subramanian, V., Plastic-Compatible Low Resistance Printable Gold Nanoparticle Conductors for Flexible Electronics. J. Electrochem. Soc. 2003, 150 G412-G417.

46. Layani, M.; Kamyshny, A.; Magdassi, S., Transparent Conductors Composed of Nanomaterials. Nanoscale 2014, 6 5581-5591.

47. Langley, D.; Giusti, G.; Mayousse, C.; Celle, C.; Bellet, D.; Simonato, J. P., Flexible Transparent Conductive Materials Based on Silver Nanowire Networks: A Review. Nanotechnology 2013, 24452001.

48. De, S.; Higgins, T.; Lyons, P. E.; Doherty, E. M.; Nirmalraj, P. N.; Blau, W. J.; Boland, J. J.; Coleman, J. N., Silver Nanowire Networks as Flexible, Transparent, Conducting Films: Extremely High Dc to Optical Conductivity Ratios. ACS Nano 2009, 3 1767-1774.

49. Scardaci, V.; Coull, R.; Lyons, P. E.; Rickard, D.; Coleman, J. N., Spray Deposition of Highly Transparent, Low-Resistance Networks of Silver Nanowires over Large Areas. Small 2011, 7 2621-2628.

50. $\quad$ Lee, S. J.; Kim, Y.-H.; Kim, J. K.; Baik, H.; Park, J. H.; Lee, J.; Nam, J.; Park, J. H.; Lee, T.-W.; Yi, G.-R.; Cho, J. H., A Roll-to-Roll Welding Process for Planarized Silver Nanowire Electrodes. Nanoscale 2014, 6 11828-34.

51. Hu, L.; Kim, H. S.; Lee, J.-Y.; Peumans, P.; Cui, Y., Scalable Coating and Properties of Transparent, Flexible, Silver Nanowire Electrodes. ACS Nano 2010, 4 2955-2963.

52. Lee, J. Y.; Connor, S. T.; Cui, Y.; Peumans, P., Solution-Processed Metal Nanowire Mesh Transparent Electrodes. Nano Lett. 2008, 8 689-692.

53. Madaria, A. R.; Kumar, A.; Ishikawa, F. N.; Zhou, C. W., Uniform, Highly Conductive, and Patterned Transparent Films of a Percolating Silver Nanowire Network on Rigid and Flexible Substrates Using a Dry Transfer Technique. Nano Research 2010, 3 564-573.

54. Sorel, S.; Bellet, D.; Coleman, J. N., Relationship between Material Properties and Transparent Heater Performance for Both Bulk-Like and Percolative Nanostructured Networks. ACS Nano 2014, 8 4805-4814.

55. Sepulveda-Mora, S. B.; Cloutier, S. G., Figures of Merit for High-Performance Transparent Electrodes Using Dip-Coated Silver Nanowire Networks. J. Nanomater. 2012, 2012286104.

56. Gysling, H. J., Nanoinks in Inkjet Metallization - Evolution of Simple Additive-Type Metal Patterning. Curr. Opin. Colloid Interface Sci. 2014, 19 155-162.

57. Wu, J.-T.; Hsu, S. L.-C.; Tsai, M.-H.; Liu, Y.-F.; Hwang, W.-S., Direct Ink-Jet Printing of Silver Nitrate-Silver Nanowire Hybrid Inks to Fabricate Silver Conductive Lines. J. Mater. Chem. 2012, 22 15599-15605.

58. Lu, H.; Lin, J.; Wu, N.; Nie, S.; Luo, Q.; Ma, C.-Q.; Cui, Z., Inkjet Printed Silver Nanowire Network as Top Electrode for Semi-Transparent Organic Photovoltaic Devices. Appl. Phys. Lett. 2015, 106093302.

59. Stegen, J., Mechanics of Carbon Nanotube Scission under Sonication. J. Chem. Phys. 2014, 140244908.

60. Derby, B.; Reis, N., Inkjet Printing of Highly Loaded Particulate Suspensions. MRS Bull. 2003, 28 815-818.

61. Lyon, P. J.; Carter, J.; Creighton, C.; Gregory, H. Solvents for Pedot-Solutions for Inkjet Printing. 2009. 
62. Castano, J.; Girones, X. Method of Reducing Vertical Banding in Ink Jet Printing. 2003. 63. Stauffer, D. S.; Aharony, A., Introduction to Percolation Theory. Taylor and Francis: London, 1994.

64. De, S.; Coleman, J. N., The Effects of Percolation in Nanostructured Transparent Conductors. MRS Bull. 2011, 36 774-781.

65. De, S.; King, P. J.; Lyons, P. E.; Khan, U.; Coleman, J. N., Size Effects and the Problem with Percolation in Nanostructured Transparent Conductors. ACS Nano 2010, 4 7064-7072.

66. Leem, D.-S.; Edwards, A.; Faist, M.; Nelson, J.; Bradley, D. D. C.; de Mello, J. C., Efficient Organic Solar Cells with Solution-Processed Silver Nanowire Electrodes. Adv. Mater. 2011, 23 4371-4375.

67. Sorel, S.; Lyons, P. E.; De, S.; Dickerson, J. C.; Coleman, J. N., The Dependence of the Optoelectrical Properties of Silver Nanowire Networks on Nanowire Length and Diameter. Nanotechnology 2012, 23185201.

68. Hecht, D.; Hu, L. B.; Gruner, G., Conductivity Scaling with Bundle Length and Diameter in Single Walled Carbon Nanotube Networks. Appl. Phys. Lett. 2006, 89.

69. Lee, P.; Lee, J.; Lee, H.; Yeo, J.; Hong, S.; Nam, K. H.; Lee, D.; Lee, S. S.; Ko, S. H., Highly Stretchable and Highly Conductive Metal Electrode by Very Long Metal Nanowire Percolation Network. Adv. Mater. 2012, 24 3326-3332.

70. Garnett, E. C.; Cai, W.; Cha, J. J.; Mahmood, F.; Connor, S. T.; Christoforo, M. G.; Cui, Y.; McGehee, M. D.; Brongersma, M. L., Self-Limited Plasmonic Welding of Silver Nanowire Junctions. Nat. Mater. 2012, 11 241-249. 
TOC Image

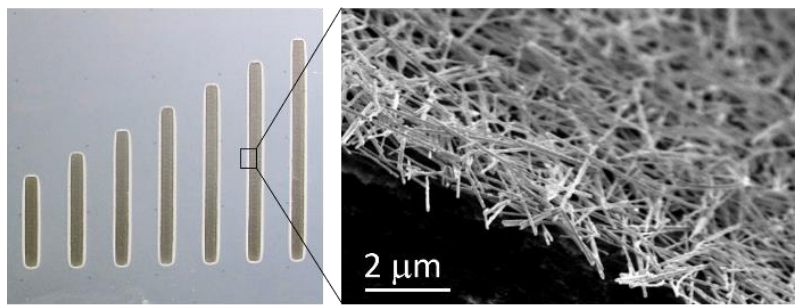

TOC Caption: We demonstrate inkjet printing of silver nanowires at relatively low temperature to form networks with high conductivity. 